\title{
The encounter of tumor and SARS-CoV-2: Pathological findings in a patient with colon cancer and COVID-19
}

\section{Weixiang Zhong}

Zhejiang University School of Medicine First Affiliated Hospital https://orcid.org/0000-0002-5493-893X Jun Li

Zhejiang University School of Medicine First Affiliated Hospital

\section{Mei Kong}

Zhejiang University School of Medicine First Affiliated Hospital

\section{Qiqi Gao}

Zhejiang University School of Medicine First Affiliated Hospital

\section{Xiaodong Teng}

Zhejiang University School of Medicine First Affiliated Hospital

Tingbo Liang ( $\square$ liangtingbo@zju.edu.cn )

Zhejiang University School of Medicine First Affiliated Hospital

\section{Jing Zhang ( $\nabla$ jzhang1989@zju.edu.cn )}

Zhejiang University School of Medicine First Affiliated Hospital

\section{Case report}

Keywords: Coronavirus disease 2019 (COVID-19), Angiotensin converting enzyme 2 (ACE2), Tumor microenvironment (TME), Colon cancer, severe acute respiratory syndrome coronavirus 2 (SARS-CoV-2)

Posted Date: July 1st, 2020

DOI: https://doi.org/10.21203/rs.3.rs-38812/v1

License: (c) (1) This work is licensed under a Creative Commons Attribution 4.0 International License. Read Full License 


\section{Abstract}

As coronavirus disease 2019 (COVID-19) spreads worldwide, there have been few reports of infections among the cancer population, although more than six million cases have been confirmed. Here we studied a surgical specimen from a patient with colon cancer and COVID-19 and two tissue microarrays comprising 103 colorectal cancer and 108 enterocyte cases pathologically. The results showed that, severe acute respiratory syndrome coronavirus 2 (SARS-CoV-2) nucleocapsid antibody was positively expressed in colon cancer tissues. Moreover, the angiotensin converting enzyme 2 (ACE2) was overexpressed in both colorectal cancer tissue microarray and tumor tissue from this patient, with a positive rate as high as $93.2 \%$. Finally, this case of SARS-CoV-2 infection in colon cancer tumor microenvironment (TME) is a failure of immune homeostasis, due to the decrease in TIA + and Granzyme $\mathrm{B}+(\mathrm{GrB}) \mathrm{CD} 8+\mathrm{T}$ cell proportion and the increase in PD-1 + CD8 + T cell proportion. In summary, we speculate that these three factors may have contributed significantly to infection risk and severity in the patient.

\section{Introduction}

As coronavirus disease 2019 (COVID-19) spreads worldwide, more than six million cases and 378,548 deaths have been reported in 215 countries and regions according to WHO statistics on 2 June 2020. Previously, statistical evidences supported that the risk of infection and adverse events in the cancer population was higher than that in non-cancer population, and attributed it to systemic immunosuppression in cancer patients.[1] However, some researchers believe that the evidence was not convincing.[2, 3] It was also found that ACE2 was highly expressed in the tumor cells by single-cell RNA sequencing. However, the histological evidence for the relationship between ACE2 overexpression in tumor cells, the microenvironment of peritumoral inflammatory cells, and COVID-19 is scarce,[4] In addition, SARS-CoV-2 infection was found in over $50 \%$ stool samples, with more than $10 \%$ patients exhibiting gastrointestinal (GI) symptoms. Particularly, the SARS-CoV-2 infection rate was $100 \%$ in esophagus, stomach, duodenum, and rectum in the endoscopic biopsies of two severe patients with $\mathrm{GI}$ symptoms. Therefore, the GI tract may be a potential transmission route and target organ of SARS-CoV-2. $[5,6]$ Recently, immunohistochemical (IHC) staining has confirmed residual SARS-CoV-2 in the pulmonary tissues of a ready-to-discharge patient.[7] Here, we described the histological evidence of tissue in-situ morphology and the TME in a patient with COVID-19 and sigmoid colon cancer.

\section{Case Report}

A 62-year-old man was given tracheal intubation for chest tightness after detecting SARS-CoV-2 using real-time (RT-PCR) in sputum samples during a week of hospitalization at a local hospital. The patient was then diagnosed with critical COVID-19 using computed tomography (CT) (Figure. 2a) after being transferred to the ICU of our hospital on 29 January 2020. On the third day, he showed diarrheal symptoms with bloody stool, which gradually improved in the following five days. Until the seventh day, the fecal occult blood test reports were negative, but respiratory difficulties continued to aggravate. After 
two weeks, the patient was provided with veno-venous extracorporeal membrane oxygenation (VV-ECMO) treatment and re-developed bloody stool the next day. Abdominal enhancement CT indicated sigmoid colon occupation (Figure. 2b) and colonoscopy suggested carcinoma. After two tests for SARS-CoV-2 nucleic acid showed negative results and the body temperature returned to normal, the patient underwent radical resection of sigmoid cancer and colostomy on 21 February 2020. Family members living with him, including his wife, son, and daughter-in-law, were released from quarantine after being tested negative for SARS-CoV-2 using nucleic acid tests. The key clinical features are summarized in Fig. 1.

Macroscopic examination showed a $6.5 \cdot 5.0 \mathrm{~cm}$ ulcerated and necrotic mass in the middle of the sigmoid colon. Histological examination revealed that the tumor surface and lumen were necrotic, presented a tubular glandular and sylph-like arrangement, and showed the infiltration of peritumor fibrotic stroma by a large number of inflammatory cells (Fig. 3a).

IHC staining showed that CK20, CDX-2, and mismatch repair marker (MSH2, MSH6, MLH1, and PMS2) expression were positive, while CK7 and CMV expression were negative in the tumor cells. Furthermore, MUC2 was negatively expressed in the tumor cells (Fig. 3b). Compared with that in the surrounding enterocytes, ACE2 expression, located in the cell membrane and cytoplasm of tumor cells, were significantly enhanced (Fig. 3c). Two tissue microarray composed of 103 carcinoma tissues and 183 enterocyte were generated to validate that ACE2 expression percentage was very high in both carcinoma and enterocytes (93.2\% and $75.4 \%$, respectively), but its intensity was higher in carcinoma than that in controls, the strongly positive ACE2 expression being $73.8 \%$ in the tumor, while being only $4.4 \%$ in the enterocytes (Table 1). In colon cancer cells, SARS-CoV-2 nucleocapsid expression was localized in the cell membrane and cytoplasm (Fig. 3d), however, all tissue microarray samples showed negative expression. CD3, CD4, CD8, CD20, CD56, CD163, TIA-1, GrB, and PD-1 expression in tumor microenvironment were immunohistochemically assessed. The results revealed that the majority of infiltrated immune cells are mononuclear phagocyte, followed by some T lymphocytes, but minimal natural killer (NK) cells infiltrated ration, which express CD163 (Fig. 4a), CD56 and CD3 (Fig. 4b) respectively. CD20 showed limited expression in the germinal center. The proportion of CD4 and CD8 positive $T$ cells was similar. The percentage of CD8 + T cells producing the cytotoxic molecule were further assessed. TIA-1, GrB (Fig. 4c), and PD-1 (Fig. 4d) were detected in a limited number of infiltrating CD8 + T cells, with TIA-1/CD8, $\mathrm{GrB} / \mathrm{CD} 8$, and PD-1/CD8 being $0.22,0.20$, and 0.34 , respectively. 
Table 1

ACE2 expression in tissue microarrays having 103 colorectal cancer and 183 enterocyte cases

\begin{tabular}{|lllll|}
\hline & Total & Colorectal carcinoma & Enterocyte & P value \\
\hline ACE2 Level & 283 & 103 & 183 & $<0.0001$ \\
\hline 0 & 51 & $7(6.8 \%)$ & $45(24.6 \%)$ & \\
\hline 1 & 150 & $20(19.4 \%)$ & $130(71.0 \%)$ & \\
\hline 2 & 84 & $76(25.2 \%)$ & $8(4.4 \%)$ & \\
\hline
\end{tabular}

\section{Discussion}

As we know, ACE2, a key receptor for SARS-CoV-2 infection, aids coronavirus attachment to membranes, entry to host cell, and replication; moreover, the attachment of the virus to cell surface ACE2 protects them from immune surveillance mechanisms.[8] Recent studies have revealed that the modified $S$ protein of SARS-CoV-2 has a significantly higher affinity for ACE2 and is 10- to 20-fold more likely to bind to ACE2 in human cells than that of the SARS-CoV.[9] This study shows that ACE2 were expressed in the cell membrane and cytoplasm of tumoral tissues, but weakly expressed in the enterocytes, which is consistent with ACE2 distribution previously reported.[10] Meanwhile, SARS-CoV-2 nucleocapsid antibody was expressed in the cell membrane and cytoplasm of tumoral tissues. Therefore, initially, we speculated that the strong expression of ACE2 in cancer cells of the patient increases the number of adhesion sites and potential viral load for SARS-CoV-2. This led to increased susceptibility to SARS-CoV-2 and increased severity of manifestations (compared with those who live with him but were not infected, particularly his wife, a 59-year-old woman). This notion is also consistent with the conclusion that transgenic mice were more susceptible to SARS-CoV than were wild-type mice.[11] Furthermore, cell-surface MUC2 is expressed in goblet cells and plays a critical role in the active defense against virus infection. We found that MUC2 was negatively expressed in the carcinoma, which had a defective MUC2 mucin barrier, which might lead to the deficiency in resistance against SARS-CoV-2 infection in the colonic mucosa.[12] Moreover, the intense expression of ACE2 in tumor cells of the patients with colon cancer could further indicate that the GI may be a potential transmission route and target organ of SARS-CoV-2.

Previous reports indicate that $C D 4+$ and $C D 8+T$ cell counts are reduced significantly in the patients with COVID-19, suggesting that SARS-CoV-2 suppresses T cell differentiation, which may be related to the severity of COVID-19.[13] Furthermore, the peripheral CD4 + and CD8 T + cell counts are substantially reduced in the blood of the patients with COVID-19, while, mononuclear inflammatory infiltrates, dominated by lymphocytes, are seen in both lungs.[14] Although infiltrated CD $4+$ and CD $8+T$ cells were observed in the carcinoma tissue, colonic carcinoma environment is a particular circumstance where the immune response could be inefficient or unresponsive, resulting in uncontrolled growth of the carcinoma cells. The activated TIA-1 + and GrB $+\mathrm{CD} 8+\mathrm{T}$ cells were limited in number in colonic carcinoma, while the PD-1 + CD8 + T cells increased, which is not only one of the signs of T cell failure, but also a common 
pathway inhibitor for CD8 T cells following respiratory virus infection.[15] Therefore, a failure of immune homeostasis occurs, which consequently causes poor reactivity in the TILs. Substantial and interactive impacts between carcinoma and immune cells create a microenvironment suitable for SARS-CoV-2 infection.

On the contrary, COVID-19 causes multiple organ dysfunction syndrome rather than respiratory failure, which might be attributable to the widespread distribution of ACE2 in multiple organs. ACE2 overexpression in colonic carcinoma might be the reason for the obvious symptom in the GI.

In summary, pathologically, we observed, for the first time, a positive expression of SARS-CoV-2 nucleocapsid antibody in colon cancer tissues. Moreover, ACE2, a key receptor for SARS-CoV-2, was overexpressed in the colon tumor cells of the patient with COVID-19 and the TME may be more suitable for SARS-CoV-2 survival. we speculate that these factors may have contributed significantly to infection risk and severity in the patient. We also provided in situ morphological evidence indicating that patients with cancer may have increased SARS-CoV-2 susceptibility and severe infection and the GI may be a potential transmission route and target organ for SARS-CoV-2.

\section{Declarations}

\section{Conflict of interests}

The authors have disclosed that they have no significant relationships with or financial interest in any commercial companies pertaining to this article.

\section{Contributors}

Weixiang Zhong and Jun Li performed most of the experiments and wrote the paper; Xiaodong Teng, Tingbo Liang and Jing Zhang designed the experiments; Mei Kong and Qiqi Gao analyzed the data and all authors approved the final paper.

\section{Acknowledgments}

Written informed consent was obtained from the patient for publication of this Case report and any accompanying images. A copy of the written consent is available for review by the Editor-in-Chief of this journal.

\section{References}

1. Liang W, Guan W, Chen R, Wang W, Li J, Xu K, Li C, Ai Q, Lu W, Liang H, et al. Cancer patients in SARSCoV-2 infection: a nationwide analysis in China. The Lancet Oncology. 2020;21(3):335-7.

2. Wang H, Zhang L. Risk of COVID-19 for patients with cancer. The Lancet Oncology 2020, 21(4). 
3. Xia Y, Jin R, Zhao J, Li W, Shen H. Risk of COVID-19 for cancer patients. The Lancet Oncology 2020, 21(4).

4. Chen H, Xuan B, Yan Y, Zhu X, Shen C, Zhao G, Ji L, Xu D, Xiong H, Yu T, et al: Profiling ACE2 expression in colon tissue of healthy adults and colorectal cancer patients by single-cell transcriptome analysis. medRxiv 2020.

5. Lin L, Jiang X, Zhang Z, Huang S, Zhang Z, Fang Z, Gu Z, Gao L, Shi H, Mai L, et al: Gastrointestinal symptoms of 95 cases with SARS-CoV-2 infection. Gut 2020.

6. Lamers MM, Beumer J, van der Vaart J, Knoops K, Puschhof J, Breugem TI, Ravelli RBG, Paul van Schayck J, Mykytyn AZ, Duimel HQ, et al: SARS-CoV-2 productively infects human gut enterocytes. Science 2020.

7. Yao XH, He ZC, Li TY, Zhang HR, Wang Y, Mou H, Guo Q, Yu SC, Ding Y, Liu X, et al: Pathological evidence for residual SARS-CoV-2 in pulmonary tissues of a ready-for-discharge patient. Cell Res 2020.

8. Brake SJ, Barnsley K, Lu W, McAlinden KD, Eapen MS, Sohal SS. Smoking Upregulates AngiotensinConverting Enzyme-2 Receptor: A Potential Adhesion Site for Novel Coronavirus SARS-CoV-2 (Covid19). J Clin Med 2020, 9(3).

9. Wrapp D, Wang N, Corbett KS, Goldsmith JA, Hsieh CL, Abiona O, Graham BS, McLellan JS. Cryo-EM structure of the 2019-nCoV spike in the prefusion conformation. Science. 2020;367(6483):1260-3.

10. Hamming I, Timens W, Bulthuis ML, Lely AT, Navis G, van Goor H. Tissue distribution of ACE2 protein, the functional receptor for SARS coronavirus. A first step in understanding SARS pathogenesis. $J$ Pathol. 2004;203(2):631-7.

11. Yang XH, Deng W, Tong Z, Liu YX, Zhang LF, Zhu H, Gao H, Huang L, Liu YL, Ma CM, et al. Mice transgenic for human angiotensin-converting enzyme 2 provide a model for SARS coronavirus infection. Comp Med. 2007;57(5):450-9.

12. Boshuizen JA, Reimerink JH, Korteland-van Male AM, van Ham VJ, Bouma J, Gerwig GJ, Koopmans MP, Buller HA, Dekker J, Einerhand AW. Homeostasis and function of goblet cells during rotavirus infection in mice. Virology. 2005;337(2):210-21.

13. Chen G, Wu D, Guo W, Cao Y, Huang D, Wang H, Wang T, Zhang X, Chen H, Yu H, et al: Clinical and immunological features of severe and moderate coronavirus disease 2019. J Clin Invest 2020.

14. Xu Z, Shi L, Wang Y, Zhang J, Huang L, Zhang C, Liu S, Zhao P, Liu H, Zhu L, et al. Pathological findings of COVID-19 associated with acute respiratory distress syndrome. Lancet Respir Med. 2020;8(4):420-2.

15. Schmidt ME, Varga SM. The CD8 T Cell Response to Respiratory Virus Infections. Front Immunol. 2018;9:678.

\section{Figures}




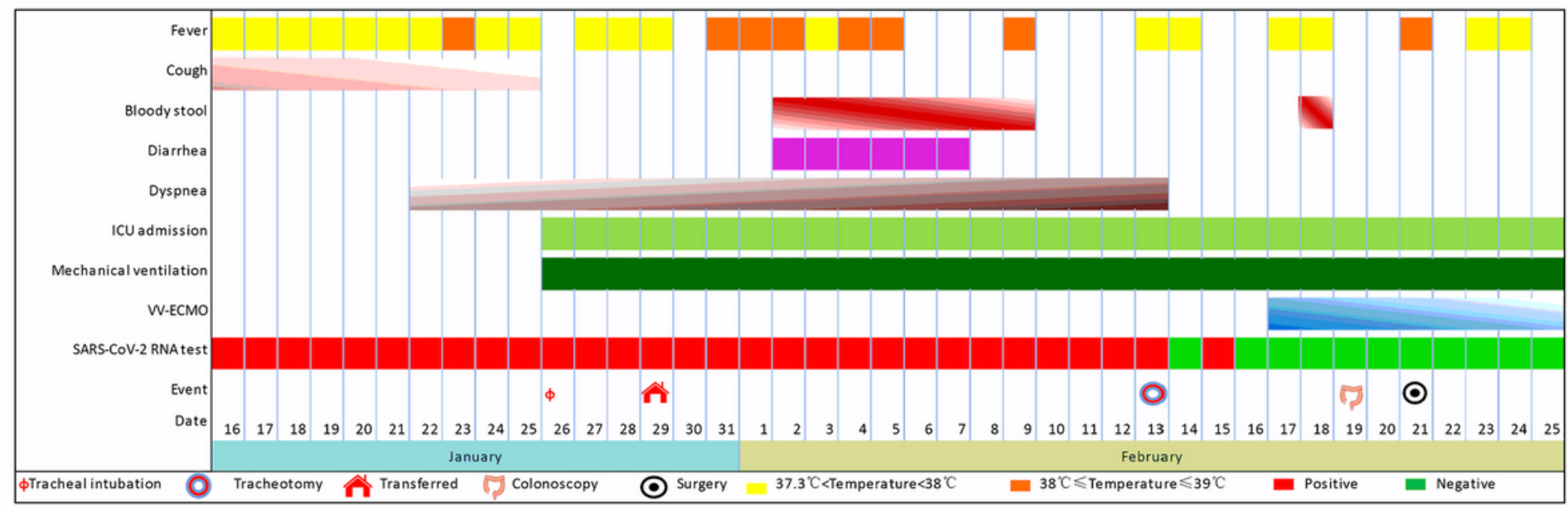

\section{Figure 1}

The clinical course of the patient with colon cancer and COVID-19. SARS-CoV-2=severe acute respiratory syndrome coronavirus 2. VV-ECMO=veno-venous extracorporeal membrane oxygenation.
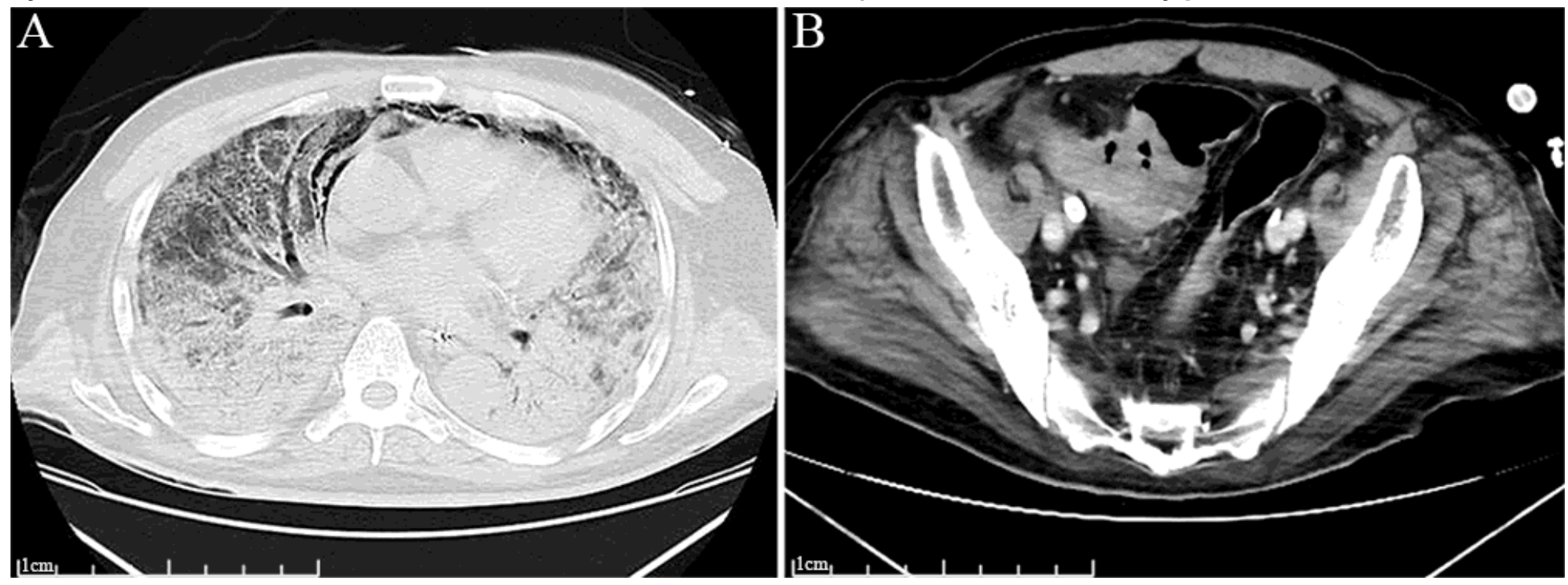

Figure 2

Transverse thin-section computed tomography (CT) scans in the patient with colon cancer and COVID-19. A. Chest CT shows diffuse ground-glass opacities in both lungs, giving a white lung appearance, with air bronchograms. B. Abdominal enhancement CT reveals that the wall of the middle sigmoid colon thickened markedly to form an ulcerated mass with marked enhancement and rough serous surface. 


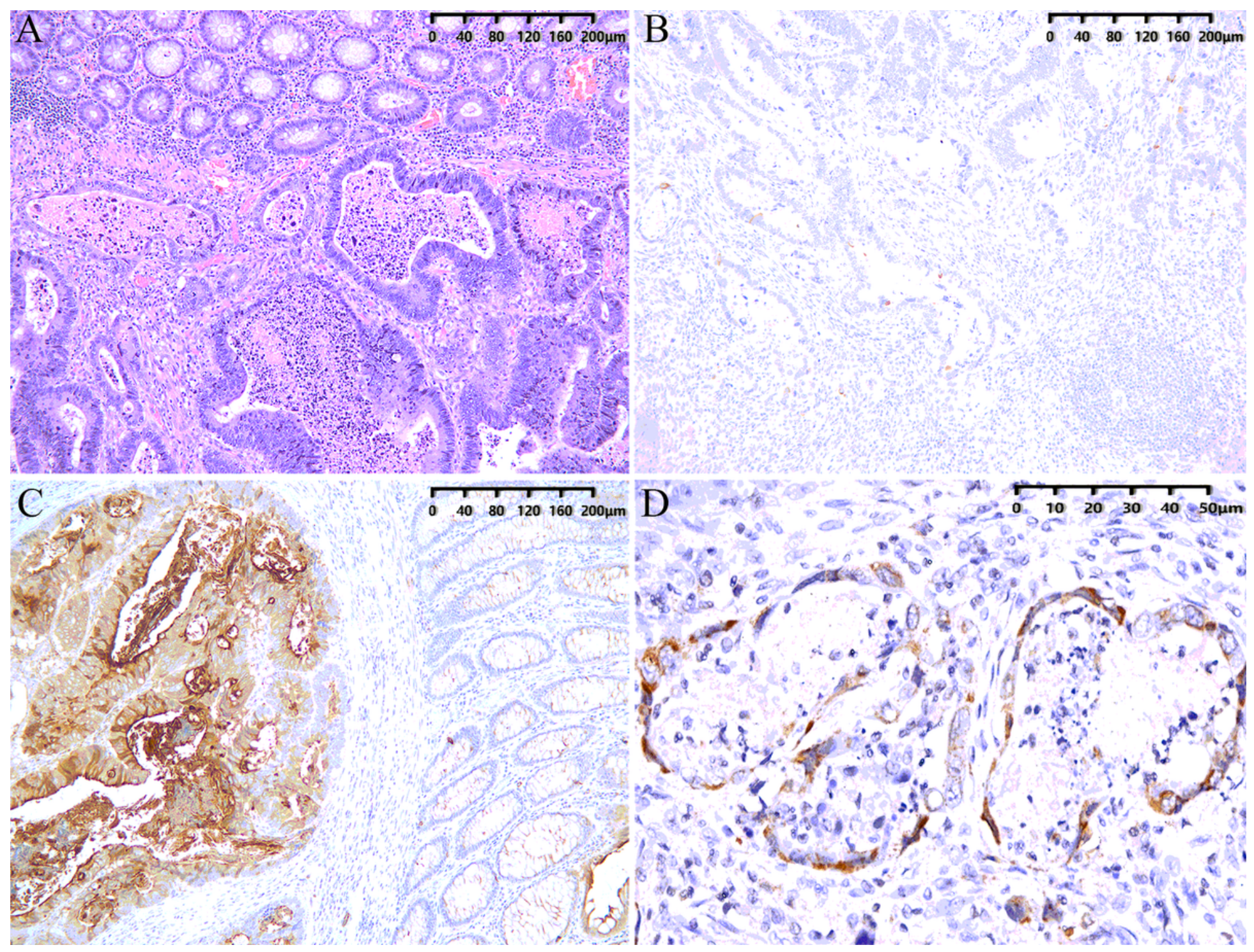

Figure 3

Pathological manifestations of the tumoral tissue. A. The tumor was arranged in a tubular gland under the mucosa, with infiltrative growth and a large number of inflammatory cells infiltrating the fibrotic stroma. B. The negative MUC2 expression in tumoral tissue. C. ACE2 expression was located in the cell membrane and cytoplasm of the tumor cells and significantly enhanced compared with that in the enterocytes. D. In colon cancer samples, SARS-CoV-2 nucleoprotein $(\mathrm{N})$ was localized in the cell membrane and cytoplasm. 


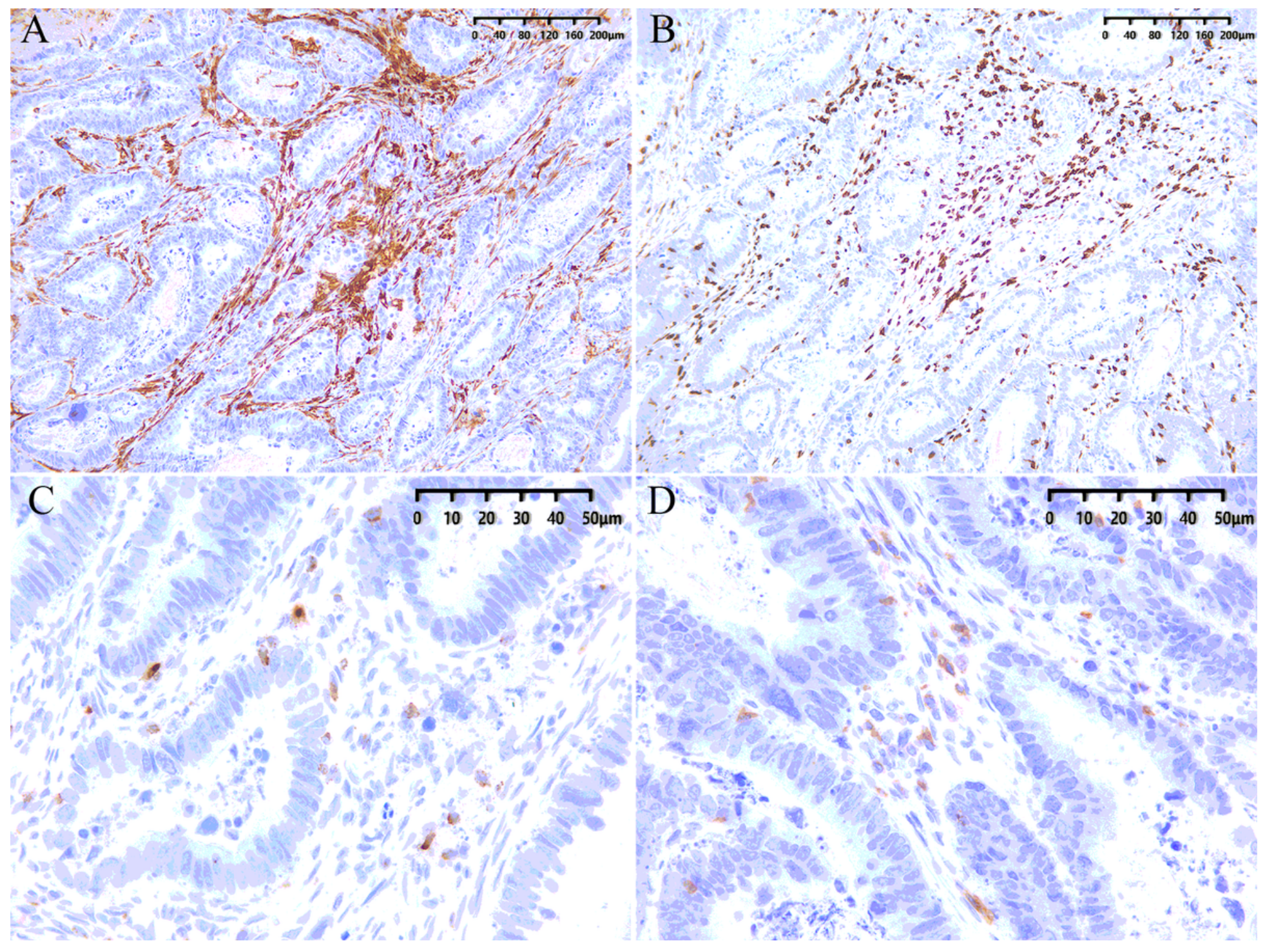

Figure 4

Phenotypes of major immune cells in the TME. A. The majority of infiltrated immune cells in TME are CD163+ mononuclear phagocytes $B$. The subsequent infiltrated immune cells in TME are CD3+ $T$ lymphocytes. C. The proportion of $\mathrm{GrB}+/ \mathrm{CD} 8+\mathrm{T}$ cell was 0.34 . D. The proportion of PD-1+/CD8+T cell was 0.34 .

\section{Supplementary Files}

This is a list of supplementary files associated with this preprint. Click to download.

- Supplementaryfile.docx 